\section{Divertículo vesical congénito en un recién nacido. Reporte de caso}

\author{
Gómez-Contreras DE, Pinzón-Uresti MA, Barragán-Albo F, Rodríguez-Balde- \\ rrama I
}

\begin{abstract}
Resumen
ANTECEDENTES: El divertículo vesical congénito es una hernia de la mucosa vesical, formada a través de las fibras musculares de la pared vesical. Es una alteración poco frecuente y extremadamente excepcional durante la infancia.
\end{abstract}

CASO CLÍNICO: Recién nacido de sexo femenino, con vómito e intolerancia a la vía oral desde las primeras horas de vida extrauterina. Durante el examen físico se percibió el abdomen globoso, con peristalsis adecuada; a la palpación un poco tenso y con ligero dolor; no se encontraron visceromegalias ni plastrones. La radiografía toracoabdominal evidenció las asas intestinales distendidas, desplazadas hacia arriba y al lado derecho; también se observó una imagen radiopaca en el hemiabdomen inferior e izquierdo. El ultrasonido abdominal demostró un defecto sacular, de bordes bien definidos, dependiente del domo vesical en contacto con el borde hepático, sugerente de divertículo vesical, que finalmente se confirmó con cistograma miccional.

CONCLUSIÓN: El divertículo vesical congénito debe considerarse como diagnóstico diferencial en pacientes pediátricos. El tratamiento conservador inicial y posteriormente con diverticulectomía y reparación definitiva primaria ofrecen resultados satisfactorios.

PALABRAS CLAVE: Congénito; divertículo vesical congénito; pared vesical; reparación.

Rev Mex Urol. 2018 Jan-Feb;78(1):40-43.

\section{Congenital bladder diverticulum in a newborn: A case report}

Gómez-Contreras DE, Pinzón-Uresti MA, Barragán-Albo F, Rodríguez-Balderrama I

\footnotetext{
Abstract

BACKGROUND: Congenital bladder diverticulum is a herniation of the bladder mucosa via the muscle fibers of the bladder wall. It is a rare entity and is uncommon in children.

CLINICAL CASE: A newborn female infant presented with vomiting and feeding intolerance within the first hours of extrauterine life. Physical examination revealed a distended abdomen with adequate peristalsis. Upon palpation, the abdomen was tense and there was slight pain, but no visceromegaly or adhesions were found. Thoracoabdominal x-ray identified distended intestinal segments, displaced
}

Servicio de Neonatología y Pediatría, Hospital Universitario Dr. José Eleuterio González, Universidad Autónoma de Nuevo León, Monterrey.

Recibido: febrero 2017

Aceptado: diciembre 2017

Correspondencia

Dra. Diana Edith Gómez Contreras

dianiz_e@hotmail.com

Este artículo debe citarse como

Gómez-Contreras DE, Pinzón-Uresti MA, BarragánAlbo F, Rodríguez-Balderrama I. Divertículo vesical congénito en un recién nacido. Reporte de caso. Rev Mex Urol. 2018 ene-feb;78(1):40-43.

DOI: https://doi.org/10.24245/revmexurol.v78i1.1270 
upwards and to the right. There was also a radio-opaque image in the lower left hemiabdomen. Abdominal ultrasound revealed a saccular defect with well-defined edges that was dependent on the bladder dome, touching the edge of the liver. The defect was suggestive of a bladder diverticulum, which was confirmed through voiding cystourethrography.

CONCLUSIONS: Congenital bladder diverticulum should be considered in the differential diagnosis in pediatric patients. Conservative management as initial treatment, with diverticulectomy and definitive primary repair performed at a later point in time, offer satisfactory results.

KEYWORDS: Congenital; Urinary bladder diverticulum; Bladder mucosa; Congenital bladder diverticula; Vesical repair.

\section{ANTECEDENTES}

El divertículo vesical congénito es una hernia de la mucosa vesical, formada a través del hiato ureteral, entre el uréter intravesical y el techo del hiato ureteral. ${ }^{1}$ Aunque su etiología es desconocida, la teoría más aceptada señala que aparece como una capa del detrusor anormalmente delgada o parcialmente desarrollada, lo que genera una protrusión de la mucosa de la vejiga a través de la pared muscular alterada. ${ }^{1,2}$ La localización más frecuente de divertículos es paraureteral en $90 \%$ y posterolateral en $10 \%$ de los casos. ${ }^{3,4}$

Los divertículos vesicales se clasifican en congénitos (primarios) y adquiridos. Los congénitos aparecen en vejigas de paredes lisas, pueden ser aislados o múltiples y no muestran obstrucción de salida de la vejiga. ${ }^{1,2}$

Los divertículos vesicales son alteraciones poco frecuentes y extremadamente excepcionales durante la infancia. Su incidencia es de $1.7 \%$ de la población pediátrica, que generalmente se somete a cistograma miccional por antecedente de infecciones de vías urinarias de repetición. ${ }^{3}$

\section{CASO CLÍNICO}

Recién nacido de sexo femenino, de término, de 3360 g, talla de 50 cm, Apgar 8-9; con adecuado control prenatal, embarazo de evolución normal, nacida por cesárea debido a desproporción cefalopélvica. Tuvo vómito de contenido alimentario e intolerancia a la vía oral desde las primeras horas de vida extrauterina, por lo que ingresó al servicio de Neonatología del Hospital Universitario Dr. José Eleuterio González para su evaluación.

Durante el examen físico se encontró despierta, con irritabilidad y adecuado reflejo de succión. El abdomen globoso, con peristalsis adecuada; a la palpación un poco tenso y con ligero dolor; no se encontraron visceromegalias ni plastrones. Se solicitó radiografía toracoabdominal, que evidenció dilatación de las asas intestinales. Se descartaron otras causas de intolerancia a la vía oral, por lo que se indicó alimentación, incrementando gradualmente su cantidad por vía enteral; sin embargo, la paciente tuvo vómito en las siguientes 24 horas. La reexploración física del abdomen continuó sin reporte de signos ni síntomas; se solicitó una nueva radiografía toracoabdominal, que mostró las asas intestinales 
distendidas, desplazadas hacia arriba y al lado derecho, además de una imagen radiopaca en el hemiabdomen inferior e izquierdo (Figura 1). El ultrasonido abdominal reportó la vejiga de $7.2 \times 5.3 \times 3.4 \mathrm{~cm}$, con volumen de $67 \mathrm{~mL}$, a expensas de un defecto sacular, con bordes bien definidos, dependiente de domo vesical en contacto con el borde hepático, sugerente de divertículo vesical (Figura 2).

El cistograma miccional evidenció la vejiga urinaria en completa reposición, aumentada de

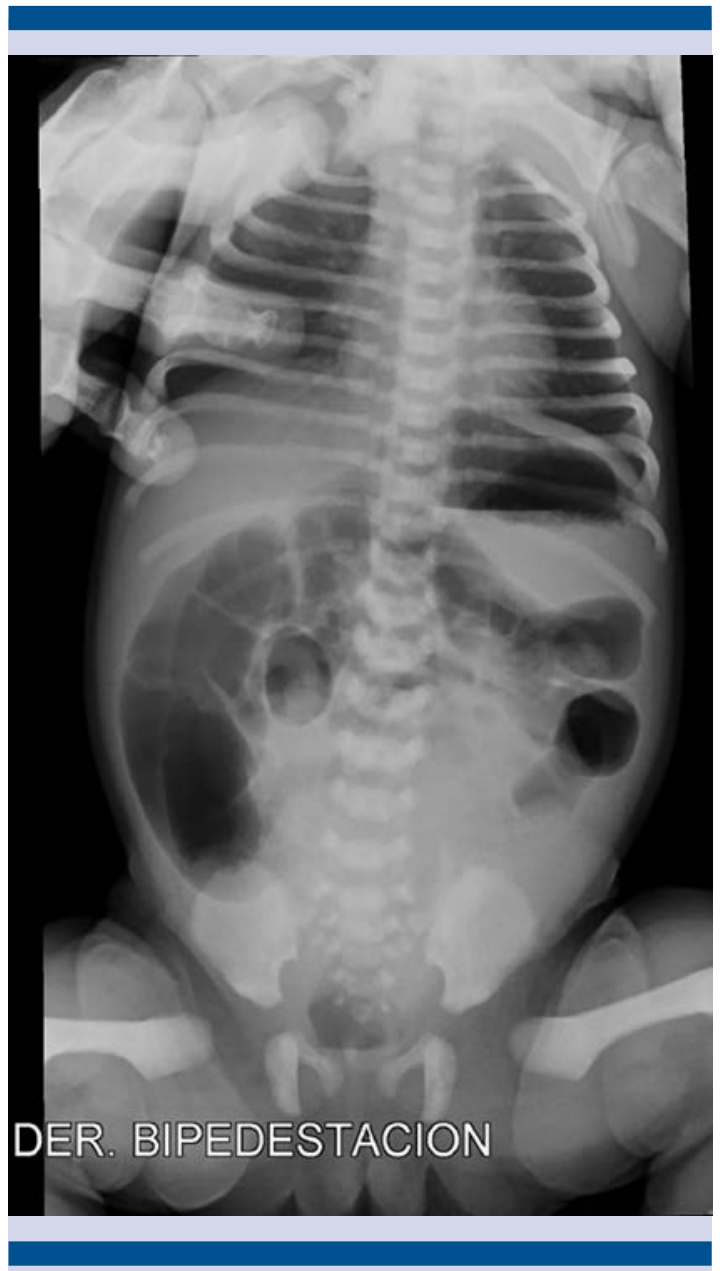

Figura 1. Radiografía toracoabdominal en bipedestación que muestra el desplazamiento de las asas intestinales hacia la derecha.

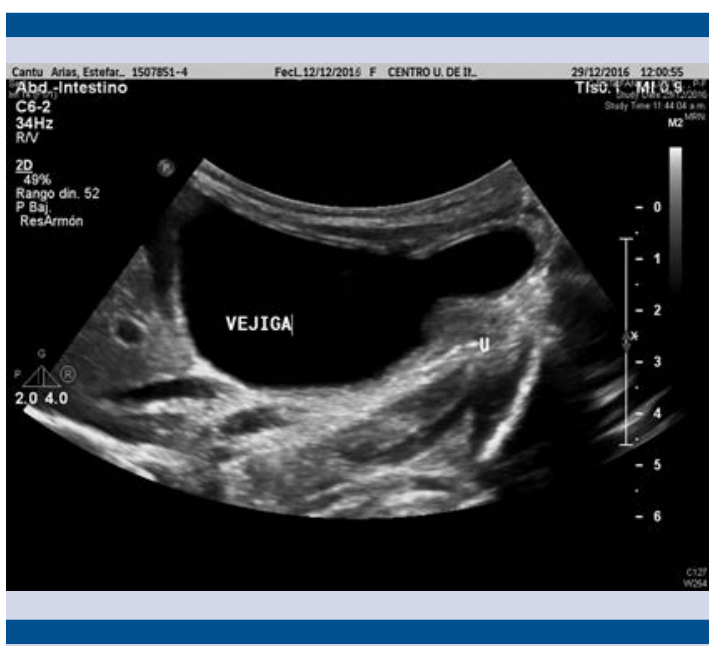

Figura 2. Ultrasonido abdominal que reporta la vejiga de $7.2 \times 5.3 \times 3.4 \mathrm{~cm}$, con volumen de $67 \mathrm{~mL}$ y el defecto sacular de bordes bien definidos.

tamaño, con imagen sacular dependiente de su domo y extensión abdominal que provocaba el desplazamiento de las asas de intestino delgado, sin defectos de llenado. De acuerdo con los hallazgos detectados se confirmó el diagnóstico de divertículo vesical (Figura 3). El examen general de orina reportó esterasas, nitritos y leucocitos positivos, por lo que se envió una muestra para urocultivo. Se inició tratamiento con antibiótico según los protocolos de la institución.

En interconsulta con el servicio de Urología Pediátrica se decidió dejar la sonda urinaria a derivación para el adecuado vaciado vesical y tratamiento conservador. Posteriormente se otorgó el alta hospitalaria y se decidió programar el procedimiento quirúrgico cuando alcance la edad óptima para la corrección definitiva de la alteración.

\section{DISCUSIÓN}

Los divertículos vesicales congénitos representan una alteración poco frecuente. ${ }^{5}$ Según la bibliografía, afectan principalmente a los hombres que 


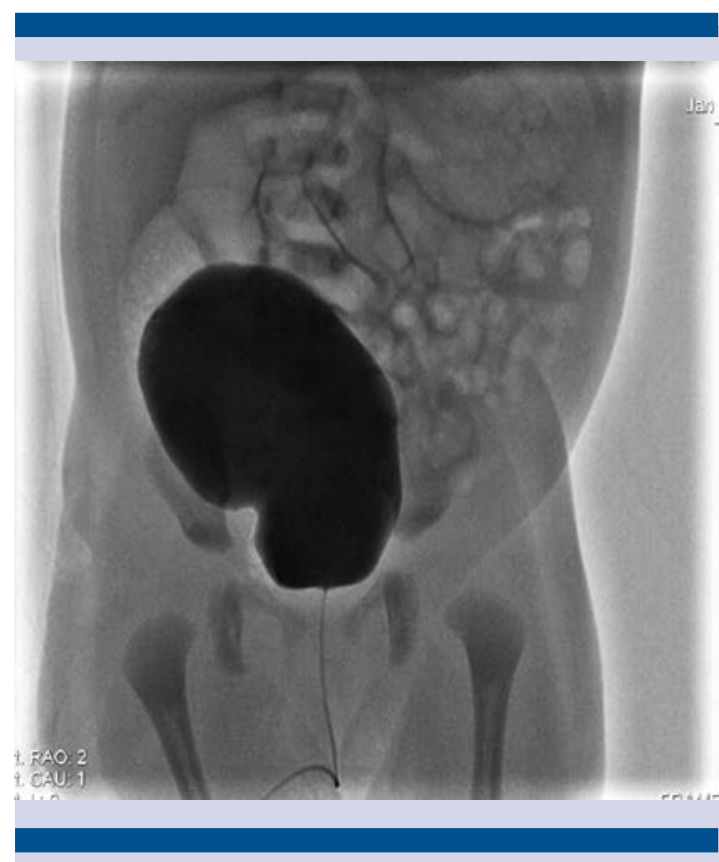

Figura 3. Cistograma miccional que evidencia la vejiga urinaria en completa repleción, aumentada de tamaño, con imagen sacular dependiente de su domo.

a las mujeres. Además del caso aquí expuesto, solo existen dos reportes de divertículo vesical en mujeres. ${ }^{3,5}$ La manifestación más común es infección de las vías urinarias u obstrucción urinaria; sin embargo, nuestra paciente inició desde las primeras horas de vida extrauterina con un cuadro de pseudooclusión intestinal, que lo convierte en un caso excepcional. ${ }^{6}$

En la paciente de este estudio, el hallazgo radiográfico más importante fue encontrar una masa radiopaca que cambiaba de posición en cada estudio de imagen; sin embargo, por medio de estos se estableció el diagnóstico definitivo. Aunque el cistograma miccional representa el estudio de referencia para establecer el diagnóstico de divertículo vesical, también pueden observarse mediante ultrasonido, tomografía computada, urograma y pielograma intravenoso. ${ }^{2}$
El tratamiento de pacientes con divertículo vesical consiste en intervención quirúrgica, principalmente diverticulectomía y reparación definitiva primaria. Los resultados suelen ser satisfactorios y con bajo índice de complicaciones; no obstante, la mayoría de los autores sugiere efectuar el procedimiento a los 6 meses de vida extrauterina, hasta completar el proceso de maduración fisiológica. ${ }^{7}$

\section{CONCLUSIÓN}

El divertículo vesical congénito es una alteración que debe considerarse como diagnóstico diferencial en neonatos e infantes de mayor edad. El cistouretrograma miccional, cuidadosamente realizado, es el estudio de referencia para establecer el diagnóstico de la alteración. El tratamiento conservador inicial y posteriormente con diverticulectomía y reparación definitiva primaria ofrecen resultados satisfactorios.

\section{REFERENCIAS}

1. Rawat J, Rashid KA, Kanojia RP, et al. Diagnosis and management of congenital bladder diverticulum in infancy and childhood: experience with nine cases at a tertiary health center in a developing country. Int Urol Nephrol 2009;41(2):237-242.

2. Psutka SP, Cendron M. Bladder diverticula in children. J Pediatr Urol 2013;9(2):129-138.

3. Garat JM, Angerri O, Caffaratti J, et al. Primary congenital bladder diverticula in children. Urology 2007;70(5):984988.

4. Singal AK, Chandrasekharam VV. Lower urinary tract obstruction secondary to congenital bladder diverticula in infants. Pediatr Surg Int 2009;25(2):1117-1121.

5. Bhat A, Bothra R, Bhat MP, et al. Congenital bladder diverticulum presenting as bladder outlet obstruction in infants and children. J Pediatr Urol 2012;8(4):348-353.

6. Shukla AR, Bellah RA, Canning DA, et al. Giant bladder diverticula causing bladder outlet obstruction in children. J Urol 2004;172(5 Pt 1):1977-1979.

7. Kok KY, Seneviratne HS, Chua HB, et al. Laparoscopic excision of congenital bladder diverticulum in a child. Surg Endosc 2000;14(5):501. 